\title{
A SIMPLE METHOD FOR DETECTING STREPTOMYCIN-RESISTANT TUBERCLE BACILLI
}

\author{
BY
}

\author{
PETER WILDY* \\ From the Department of Bacteriology, St. Thomas's Hospital Medical School, London
}

(RECEIVED FOR PUblication APRIL 18, 1951)

Since the advent of streptomycin therapy in tuberculosis, the need has arisen for a rapid, simple method for detecting the emergence of resistant strains of $M y c o$. tuberculosis. Methods so far described, for example, that of the M.R.C. (1948), are mostly designed for accuracy, but they have the disadvantage of requiring considerable manipulation. The slide culture technique (Giammalvo, Natsios, and Elton, 1949) does give quick and apparently accurate results, but is of limited application when applied to pathological material, since large numbers of organisms must be present in the specimen. Beyond the recent work of Tinne and Henderson (1950) little attention seems to have been paid to diffusion methods such as the ditch plate. For these reasons a study has been made of the factors which affect the estimation of the sensitivity of Myco. tuberculosis to streptomycin, employing a diffusion gradient in Löwenstein's medium. As a result a simple and practicable method has been evolved, which is essentially a modification of that described by Tinne and Henderson.

\section{Material and Methods}

Source of Strains. - The strains of Myco. tuberculosis had all been recently isolated from patients in St. Thomas's Hospital and the King George V Sanatorium, Hydestile, Surrey.

Sensitivity Tests in Fluid Medium.-Except that fourfold instead of doubling dilutions of the antibiotic were used, all the strains used were tested for their sensitivity to streptomycin by the technique described by the Medical Research Council (1948).

Inocula.-Inocula were regularly taken from a 14-day-old culture in Dubos's fluid medium.

\section{Preliminary Experiments}

To discover whether Löwenstein's medium could be used in a diffusion method for determining the sensitivity of Myco. tuberculosis to streptomycin, experiments were made using ditch plates prepared as follows.

Amounts, each of $30 \mathrm{ml}$., of Löwenstein-Jensen medium were inspissated in 9-cm. Petri dishes at $80^{\circ} \mathrm{C}$. for three hours. After drying, a ditch was cut on one side of the plate and filled with $2 \%$ agar containing streptomycin. Following an interval to allow the streptomycin to diffuse through the medium, the plates were inoculated by streaking the strains to be tested across the plate at right-angles to the ditch. The plates were then sealed with paraffin wax and incubated at $37^{\circ} \mathrm{C}$. for three or four weeks to find

* Working with grants from the Michael and Sidney Herbert and Leonard S. Dudgeon Funds, St. Thomas's Hospital Medical School. 
(1) the optimum concentration of streptomycin to use in the " ditch," (2) whether or not diffusion occurs uniformly, (3) the optimum period to allow for diffusion to take place, (4) the effest, if any, imposed by time of incubation, (5) the effect of size of inoculum, and (6) methods of combating the high incidence of contamination which occurred.

(1) Plates containing ditches with six different concentrations of streptomycin $(2,000$, $750,250,125,62,31 \gamma / \mathrm{ml}$.) were made. The plates were immediately inoculated with six strains of Myco. tuberculosis chosen because they had been shown by the dilution method to have differing degrees of resistance to streptomycin.

Streaks of confluent growth became visible after 14 days. They were separated from the ditch by zones of inhibition of varying lengths, and all showed a sharp end-point.

The most satisfactory differentiation between sensitive and insensitive strains was obtained on plates containing 62 and $125 \gamma / \mathrm{ml}$. streptomycin in the ditch. For this reason, the concentration used in the ditch in all subsequent experiments was $100 \gamma / \mathrm{ml}$. streptomycin.

(2) Because Löwenstein's medium is made by inspissation, it is possible that the diffusion of a substance such as streptomycin might vary considerably from place to place in the same plate. This would seem improbable in view of the following experiment.

Löwenstein's medium, $35 \mathrm{ml}$., was inspissated in a 100-ml. screw-capped medical flat bottle laid on its side. Condensation water was removed with a sterile pipette, and the bottle laid on a horizontal surface with the medium in the vertical plane. Then $10 \mathrm{ml}$. of $2 \%$ agar containing $100 \mathrm{\gamma} / \mathrm{ml}$. streptomycin was run in and allowed to set. A 14-day culture of the strain H 37 in Dubos's fluid medium was diluted 1:10 in sterile normal saline and $1 \mathrm{ml}$. flooded over the surface of the medium. Even distribution of inoculum was ensured by rocking the bottle. The bottle was turned on its face and excess fluid removed with a pipette.

After incubation for four weeks, growth had taken place up to a straight line running parallel with the edge of the streptomycin containing agar, indicating that there had been uniform diffusion of streptomycin through the medium.

(3) To determine the optimum time to allow for diffusion to take place, six plates were made from a single batch of medium. They were divided into three pairs, the ditches of which were filled five days, two days, and immediately before inoculation. During the period allowed for diffusion to take place, the plates were stored at room temperature.

TABLE 1

Relation of Time for Diffusion to ZoNe of INHibition

\begin{tabular}{|c|c|c|c|c|c|}
\hline \multicolumn{2}{|c|}{ Diffusion Time (in days) : } & $\mathbf{0}$ & 2 & 5 & \\
\hline Strain & $\begin{array}{r}\text { H } 37 \\
711 \\
681\end{array}$ & $\begin{array}{l}9 \\
4 \\
0\end{array}$ & $\begin{array}{r}18 \\
8 \\
0\end{array}$ & $\begin{array}{r}21 \\
10 \\
0\end{array}$ & $\begin{array}{l}\text { Zone of inhibi- } \\
\text { tion (in } \mathrm{mm} \text {.) }\end{array}$ \\
\hline
\end{tabular}

After incubation the zones of inhibition were measured (Table I), and it is obvious that better differentiation was obtained by allowing a period for diffusion of streptomycin to take place before inoculation. For this reason, plates have been kept for three days at bench temperature.

(4) Having established that satisfactory results could be obtained by using $100 \mathrm{\gamma} / \mathrm{ml}$. streptomycin in the ditch and by allowing a period of three days for diffusion to take place, experiments were made to see if the zone of inhibition decreased in length as 
incubation was prolonged. While this did occur, the zones of inhibition measured at two, three, and four weeks tending to recede by amounts varying from 0 to $4 \mathrm{~mm}$., in no case did the essential pattern change.

(5) Because the apparent resistance to streptomycin of Myco. tuberculosis is known to increase with the size of the inoculum (Smith and Waksman, 1947) experiments were made to determine the extent to which the size of inoculum affects the results obtained using ditch plates. For this purpose, serial fivefold dilutions of fluid cultures of seven strains were made, and a standard loopful of each dilution was streaked across the plate at right-angles to the ditch and the zones of inhibition measured after three weeks. The results obtained are shown in Table II.

TABLE II

Relation of Inoculum Size to Zone of Inhibition (mm.) With and Without $100 \mathrm{U} . / \mathrm{ML}$. Crystalline Penicillin G in the Medium

\begin{tabular}{|c|c|c|c|c|c|c|c|c|c|}
\hline \multirow{2}{*}{\multicolumn{2}{|c|}{ Dilution of Inoculum: }} & \multicolumn{8}{|c|}{ Löwenstein's Medium } \\
\hline & & $1: 1$ & $1: 5$ & $1: 25$ & $1: 125$ & $1: 625$ & $1: 3125$ & $1: 15625$ & $1: 78125$ \\
\hline Strain & $\begin{array}{l}12 C \\
33 C \\
45 C \\
47 C \\
55 C \\
80 C \\
20 C\end{array}$ & $\begin{array}{r}20 \\
0 \\
20 \\
17 \\
10 \\
0 \\
23\end{array}$ & $\begin{array}{r}20 \\
0 \\
23 \\
20 \\
11 \\
9 \\
24\end{array}$ & $\begin{array}{r}22 \\
0 \\
25 \\
22 \\
11 \\
15 \\
24\end{array}$ & $\begin{array}{l}24 \\
0 \\
25 \\
23 \\
\text { Cont } \\
15 \\
28\end{array}$ & $\begin{array}{c}26 \\
0 \\
25 \\
23 \\
\text { ninated } \\
23 \\
28\end{array}$ & $\begin{array}{r}26 \\
0 \\
26 \\
25 \\
-\overline{31}\end{array}$ & $\begin{array}{l}30 \\
10 \\
26 \\
25 \\
-31\end{array}$ & $\begin{array}{r}-0 \\
27 \\
30 \\
- \\
-\end{array}$ \\
\hline
\end{tabular}

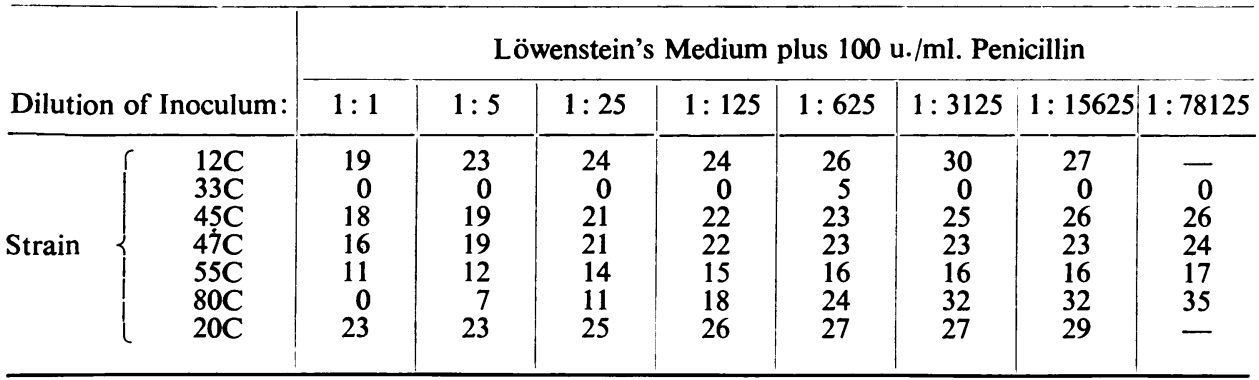

It is evident that when a strain is highly resistant the size of inoculum is immaterial, but with strains that are less resistant there is a tendency for the zone of inhibition to lengthen as the inoculum is decreased. With some resistant strains $(80 \mathrm{C})$, however, this effect is particularly marked. It is therefore clear that the size of inoculum should be controlled as far as possible. Examination of 40 consecutive recently isolated strains of varying degrees of sensitivity confirmed these findings.

(6) There was a high incidence of contamination in the above experiments due to the amount of manipulation required. This was in part reduced by replacement of the original lids at the time of inoculation with dry, sterile lids, and partly by inspissating the plates with a "false ditch" made of $4 \%$ agar. This is easily removed and quickly replaced by the ditch containing streptomycin when the medium has solidified. It was also found that if crystalline penicillin $G$ at a concentration of $100 \mathrm{u}$. $/ \mathrm{ml}$. were incorporated in the medium, contamination was virtually abolished but with no apparent alteration in the sensitivity of the strains. The results also given in Table II were obtained 
with plates which were treated in exactly the same way as those in the upper half of the Table but which contained $100 \mathrm{u}$./ml. penicillin.

Thus, although ditch plates give satisfactory results, they possess certain obvious disadvantages in that they involve much preparation and are extravagant of medium. A simple method was therefore evolved in which the vertical diffusion principle of Tinne and Henderson (1950) was adopted.

\section{A Diffusion Method Using 1-oz. Bottles}

Amounts of Löwenstein's medium, each of $5 \mathrm{ml}$., are inspissated in 1-oz. screw-capped bottles which are laid flat so that a reasonably constant depth of medium is achieved. Condensation water is removed with a sterile pipette. Then $1 \mathrm{ml}$. of $2 \%$ agar containing $100 \mathrm{\gamma} / \mathrm{ml}$. streptomycin is introduced and allowed to set on the bottom of the bottle as shown in Fig. 1. From this point forward, the bottle is kept vertical and any

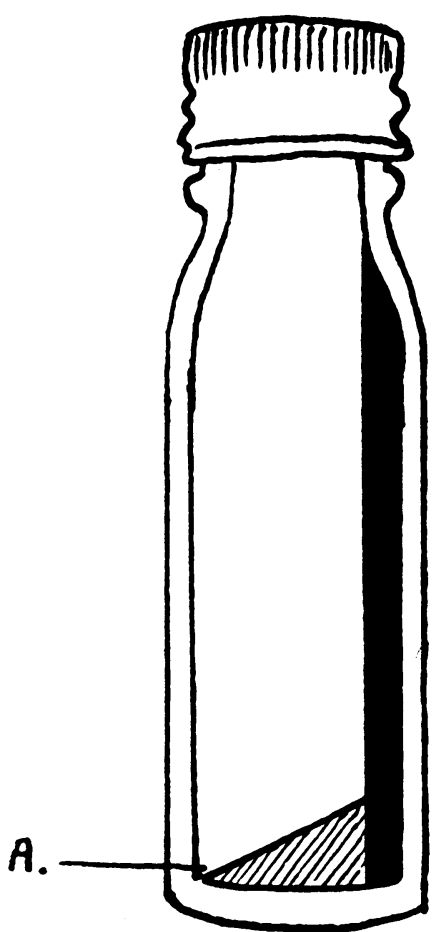

FIG. 1.-Drawing to show the method of using $1-\mathrm{oz}$. bottles for detecting strains of Myco. tuberculosis resistant to streptomycin. Condensation water collects at point $A$. Löwenstein's medium = black. Streptomycin "ditch" shaded. condensation water which may develop collects at point A between the wall of the bottle and the agar and does not interfere with the reading of the results. Inoculation is made by running one drop of a 14-day culture in Dubos's fluid medium of average turbidity from a " 50 dropper" pipette down the surface of the medium. It is not difficult to inoculate each bottle with an unknown and a control strain. When a direct test is made using pathological material, the concentrate obtained by using Petroff's method is inoculated in two dilutions, $1: 1$ and $1: 10$. In this way a rough indication of the bacterial density of the inoculum is obtained.

Whilst it is clear that most of the points covered in the preliminary experiments are applicable to the method just described, certain details were further investigated using 1-oz. bottles.

To find the best time to inoculate the "diffusion bottles" after adding the streptomycin, streptomycin agar was added to bottles containing Löwenstein's medium on five consecutive days. Or the fifth day all the bottles were inoculated with one drop of a 14-day culture of a sensitive strain of Myco tuberculosis in Dubos's fluid medium.

It was found that the length of the zones of inhibition were most regular and the end-points obtained were most distinct where five days had elapsed between the introduction of streptomycin and the time of inoculation. However, satisfactory results were obtained if the bottles were inoculated immediately after adding the streptomycin, and it has been found more convenient to follow this practice.

Because it was thought that the diffusion of streptomycin might be influenced by the slope of the " ditch," 15 bottles were made whose ditches were allowed to set with the long axes of the bottles making various angles with the horizontal plane. All were inoculated in the same way. The results observed indicated that any variation in the rate of diffusion of streptomycin due to the angle of the ditch is not sufficient to interfere with results. 


\section{Comparison of Results Obtained by the Dilution and Diffusion Bottle Methods}

Parallel determinations of the sensitivity to streptomycin of 49 strains of Myco. tuberculosis were made using the M.R.C. dilution method and diffusion bottles. In every case the same inoculating culture was used for both methods. All the inoculating cultures were of similar turbidity, and the volume applied to each bottle

TABLE III

Comparison of the Results of Parallel Determinations of Streptomycin Sensitivity of 49 Strains using Dilution and Diffusion Methods

\begin{tabular}{|c|c|c|c|c|c|c|}
\hline \multirow{2}{*}{$\begin{array}{l}\text { Length of Zone of } \\
\text { Inhibition Relative } \\
\text { to that of } \mathrm{H} 37 \text { in } \\
\text { Diffusion Bottles }\end{array}$} & \multicolumn{5}{|c|}{$\begin{array}{c}\text { Resistance Relative to } \mathrm{H} 37 \text { by Dilution } \\
\text { Method }\end{array}$} & \multirow{2}{*}{$\begin{array}{l}\text { Total } \\
\text { No. } \\
\text { of } \\
\text { Strains }\end{array}$} \\
\hline & $<1$ & 4 & 16 & 67 & $256<$ & \\
\hline $\begin{array}{c}0 \\
<\frac{1}{4} \\
\frac{1}{4}-\frac{1}{2} \\
\frac{1}{2}-\frac{3}{4} \\
\frac{3}{4}-1 \\
1<\end{array}$ & $\begin{array}{r}1 \\
-4 \\
16 \\
4\end{array}$ & $\begin{array}{l}- \\
-1 \\
1 \\
2 \\
-\end{array}$ & $\begin{array}{l}1 \\
1 \\
= \\
-\end{array}$ & $\begin{array}{l}-\frac{1}{3} \\
1 \\
1 \\
-\end{array}$ & $\begin{array}{l}7 \\
5 \\
1 \\
- \\
-\end{array}$ & $\begin{array}{r}9 \\
9 \\
3 \\
5 \\
19 \\
4\end{array}$ \\
\hline Total no. of strains & 25 & 4 & 2 & 5 & 13 & 49 \\
\hline
\end{tabular}

was controlled by using the same dropping pipette for both methods. The results are given in Table III relative to those derived from $\mathrm{H}$ 37. In the case of the diffusion method the ratio

$$
\frac{\text { zone of inhibition of test strain }}{\text { zone of inhibition of } \mathrm{H} 37}
$$

is employed to record the sensitivity of the strain under test. Whilst this is a convenient form in which to record results, it must not be inferred that there necessarily exists any simple relationship with the ratio

$$
\frac{\text { minimum concentration streptomycin inhibiting test strain }}{\text { minimum concentration streptomycin inhibiting } \mathbf{H} 37}
$$

which is used to express the results derived from the dilution method. Nevertheless, it is clear that in most cases the results obtained by both methods agree. In two instances, however, the results obtained by the two methods were at variance, one strain being apparently resistant to streptomycin by the diffusion method and sensitive by the dilution method, the other strain behaving conversely. These two estimations have not been repeated, and no explanation can therefore be given for these discrepancies.

\section{Discussion}

Using diffusion gradients of streptomycin in Löwenstein's medium, two methods for determining the resistance of Myco. tuberculosis have been evolved.

The method using 1-oz. bottles is simple and rapid. It gives a result when applied directly to pathological material in about four weeks and can be carried out in any small laboratory. Secondly, because it may be applied directly to pathological material, it is likely to give a more faithful representation of the resistance to streptomycin of the organisms in the host than methods in which one or more 
subcultures have to be made. Both these advantages are shared by the method described by Holt and Cruickshank (1949) in which parallel cultures are made on a number of Löwenstein slopes containing different concentrations of streptomycin.

Nevertheless, there are a number of disadvantages. The method is, at best, an approximate one. But this is little handicap, because the important consideration is whether the strain in question has, or has not, developed sufficient resistance for streptomycin treatment to be stopped. A more important disadvantage lies in the necessity for controlling the size of the inoculum. Tinne and Henderson (1950) make no mention of attempting to control this factor, apart from using a standard loop, but something more than this is required. Although the error entailed by neglecting this factor is usually not great if the test is regarded as approximate, there are certain strains in which the result is markedly affected by the size of the inoculum. With these strains, it is important to know the results obtained with both large and small inoculum.

Quite another use can be made of the diffusion gradient in the form of a streptomycin ditch in a Löwenstein plate. Here all the advantages inherent in ditch-plate techniques are available.

In the course of the above experiments three strains were found in which the apparent sensitivity was dependent upon the size of inoculum. With all three strains the large inocula grew up to the ditch containing $100 \gamma / \mathrm{ml}$. streptomycin, but the small inocula showed zones of inhibition of from 20 to $30 \mathrm{~mm}$. Behaviour of this kind is similar to that obtained on penicillin ditch plates with staphylococci which produce penicillinase (Barber, 1947). Whereas it is possible that these strains produce a substance antagonistic to streptomycin, the same picture might be obtained with either a mixture of sensitive and resistant strains or with an exaggeration of the variation in sensitivity to streptomycin on the part of individual cells described by Pyle (1947). These three strains are being further investigated with these points in mind.

In conclusion, it appears that methods employing diffusion gradients of streptomycin in Löwenstein's medium have many applications both as a simple method of detecting the emergence of resistance to streptomycin in Myco. tuberculosis and in the more detailed study of the behaviour of resistant strains.

\section{Summary}

An account is given of various factors affecting estimations of the sensitivity of Myco. tuberculosis to streptomycin by means of diffusion techniques.

A simple method which can be applied directly to pathological material is described.

My thanks are due to Professor Ronald Hare for his helpful criticism and to Mrs. D. N. Twort for technical assistance.

Barber, M. (1947). J. Path. Bact., 59, 373.

REFERENCES

Giammalvo, J. T., Natsios, G. A., and Elton, N. W. (1949). Amer. J. clin. Path., 19, 76.

Holt, H. D., and Cruickshank, R. (1949). Mon. Bull. Min. Hlth, 8, 103.

Medical Research Council (1948). Lancet, 2, 862.

Pyle, M. M. (1947). Proc. Mayo Clin., 22, 465.

Smith, D. G., and Waksman, S. A. (1947). J. Bact., 54, 67.

Tinne, J. E., and Henderson, J. L. (1950). Lancet 2, 901. 